\title{
Cooperative Peer Groups in NICE
}

\author{
Seungjoon Lee Rob Sherwood Bobby Bhattacharjee \\ Department of Computer Science, University of Maryland, College Park, Maryland \\ $\{$ slee, capveg, bobby\}@cs.umd.edu.
}

\begin{abstract}
We present a distributed scheme for trust inference in peer-to-peer networks. Our work is in context of the NICE system, which is a platform for implementing cooperative applications over the Internet. We describe a technique for efficiently storing user reputation information in a completely decentralized manner, and show how this information can be used to efficiently identify non-cooperative users in NICE. We present a simulationbased study of our algorithms, in which we show our scheme scales to thousands of users using modest amounts of storage, processing, and bandwidth at any individual node. Lastly, we show that our scheme is robust and can form cooperative groups in systems where the vast majority of users are malicious.
\end{abstract}

\section{INTRODUCTION}

NICE $^{1}$ is a platform for implementing cooperative applications over the Internet. We define a cooperative application as one that allocates a subset of its resources, typically processing, bandwidth, and storage, for use by other peers in the application. We believe a large class of applications, including on-line media streaming applications, multi-party conferencing applications, and emerging peer-to-peer applications, can all significantly benefit from a cooperative infrastructure. However, cooperative systems perform best if all users do, in fact, cooperate and provide their share of resources to the system. In this paper, we present techniques for identifying cooperative and non-cooperative users. Using our schemes, individual users can assign and infer "trust" values for other users. The inferred trust values represent how likely a user considers other users to be cooperative, and are used to price resources in the NICE system.

We focus on distributed solutions for the trust inference problem. We decompose the distributed trust inference problem into two parts: a local trust inference component that requires trust information between principals in the system as input and a distributed search component that efficiently gathers this individual trust information to be used as input for local inference algorithms. There already exist systems, e.g. e-bay, that have a centralized user-evaluation system. Other resource bartering systems, e.g. MojoNation, have also implemented centralized trust inference solutions. Our goal is to enable open applications where users do not have to register with an authority to be a part of the system. Centralized solutions do not scale in open systems, since malicious users can overwhelm the central "trust" server with spurious transactions. The most widely used decentralized trust inference scheme is probably the PGP web of trust [18], which allows

\footnotetext{
${ }^{1} \mathrm{NICE}$ is a recursive acronym for "NICE is the Internet Cooperative Environment" (See http://www.cs.umd.edu/projects/nice).
}

one level of inference. We present a new decentralized trust inference scheme that can be used to infer across arbitrary levels of trust. There is no trust-third-party or centralized repository of trust information in our scheme. Users in our system only store information they explicitly can use for their own benefit. We show that our algorithms scale well even with limited amount of storage at each node, and can be used to efficiently implement large distributed applications without involving explicit authorities. Further, our solutions allow individual users to compute local trust values for other users using their own inference algorithm of choice, and thus can be used to implement a variety of different policies.

\section{A. Cooperative Systems}

The notion of a cooperative system is not unique in networking; in fact, packet forwarding in the Internet is a cooperative venture that utilizes shared resources at routers. Our overall goal in NICE is to extend this notion to include end-applications and provide an incentive-based framework for implementing large distributed applications in a cooperative manner. Clearly, an immense amount of distributed resources can be harvested over the Internet in a cooperative manner. This observation is key in the recent surge of peer-to-peer (p2p) applications, and we believe the next generation of such p2p applications will be based upon the notions of cooperative distributed resource sharing.

A number of interesting distributed algorithms for $\mathrm{p} 2 \mathrm{p}$ systems, most notably in the area of distributed resource location, have recently been introduced. All of these schemes, however, assume that all peers in the system implicitly cooperate and implement the underlying protocols perfectly, even though it may not be explicitly beneficial to do so. Consider the following examples:

- In Gnutella [1], peers forward queries flooded on behalf of other users in the system. Each forwarded message consumes bandwidth and processing at each node it visits.

- In Chord [15], a document is "mapped" to a particular node using a hash function. Thus, a peer serves a document that is, in fact, owned by some other node in the system. Thus, peers in the system expend their own resources to serve documents for other nodes in the system. This situation is not unique to Chord; all hash-based location systems, including CAN [11], Bayeux [17], Pastry [13], have this property. It is possible to build a system in which nodes only serve a pointer to the document data and also to implement various load balancing schemes; however, even in the best load-balanced system, there can 
be temporary overloads when a large amount of local resources are expended due to external serving.

- A number of relay-based streaming media protocols have been developed and demonstrated. In these protocols, nodes devote resources such as access bandwidth for serving their child nodes.

In each example above, any individual user may choose not to devote local resources to external requests, and still get full benefit from the system. On the other hand, the integrity and correct functioning of the system depends on each user implementing the entire distributed protocol correctly and selflessly. However, experience with deployed systems, such as Gnutella and previously Napster, show that only a small subset of peers offer such selfless service to the community, while the vast majority of users use the services offered by this generous minority [4]. The goal of this work is to efficiently locate the generous minority, and form a clique of users all of whom offer local services to the community.

\section{B. Model}

In this paper, we assume that a (p2p) system can be decomposed into a set of of two-party transactions. A single transaction can be a relatively light-weight operation such as forwarding a Gnutella query or a potentially resource intensive operation such as hosting a Chord document. Next, we assume that the system consists of a set of "good" nodes that always implement the underlying protocols correctly and entirely, i.e. good users always fulfill their end of a transaction. The goal of our work is develop algorithms that will allow "good" users to identify other "good" users, and thus, enable robust cooperative groups. These are peer groups in which, with high probability, each participant successfully completes their end of each transaction. Specifically, we propose a family of distributed algorithms which can be used by users to calculate a per-user "trust" value. The trust value for node B at a node $\mathrm{A}$ is a measure of how likely node A believes a transaction with node B will be successful. In our system, users store a limited amount of information about how much other users trust them, and we present algorithms for choosing what information to store and how to retrieve this trust information. Once relevant information has been gathered, individual users may use different local inference algorithms to compute trust values.

It is important to note that we assume that good nodes are able to ascertain when a transaction is successful. Clearly, in many cases, it is not possible to efficiently determine whether a transaction fails (e.g. when a node sometimes does not serve Chord documents that it hosts). It is even more difficult to determine whether a transaction fails because of a system failure or because of non-cooperative users. For example, consider the case when all users are cooperative but a document cannot be served due to a network failure. We believe this problem is inherent in any trust-inference system that is based on transaction "quality". We discuss different policies for assigning values to transaction quality in Section IV.
The overall goal of this work is to identify cooperative users. An ideal trust inference system would, in one pass, be able to classify all users into cooperative or non-cooperative classes with no errors. However, this is not possible in practice because non-cooperative users may start out as cooperative users. The specific goals of our work are as follows:

- Let the "good" nodes find each other quickly and efficiently: Good nodes should be able to locate other good nodes without losing a large amount of resources interacting with malicious nodes. This will allow NICE to rapidly form robust cooperative groups.

- Malicious nodes and cliques should not be able to break up cooperating groups by spreading mis-information to good nodes. Specifically, we want to develop protocols in which malicious nodes are rapidly pruned out of cooperative groups. Further, we assume malicious nodes can disseminate arbitrary trust information, and the cliques formed of good nodes should be robust against this form of attack.

In Section IV, we describe algorithms that achieve our goals with low run-time overhead, both in terms of processing and network bandwidth usage. We believe this algorithm is the first practical, robust, trust inference scheme that can be used to implement large cooperative applications.

The rest of this paper is structured as follows: in the next section, we discuss prior work in distributed trust computations. In Section III, we present an overview of the NICE system, and describe how distributed trust computations are used by NICE nodes. We describe our algorithms and local node policies in Section IV, present simulation results in Section V, and conclude in Section VI.

\section{RELATED WORK}

In this section, we discuss prior work in trust inference and present a brief overview of systems that are based on notions of trust and incentive.

The concept of "trust" in distributed systems is formalized in [9] using social properties of trust. This work considers an agent's own experience to obtain [-1, 1]-valued trust, but does not infer trust across agents. Abdul-Rahman et al. [2] describe a trust model that deals with direct experience and reputational information. This model can be used, as is, in NICE to infer trust. Yu et al. [16] propose a way to compute a real-valued trust in $[-1,1]$ range from direct interactions with other agents. A product of trust values is used for reputation computation, and undesirable agents is avoided by having an observer of bad transactions disseminate information about the bad agent throughout the network. This work is primarily about using social mechanisms for regulating users in electronic communities, and the techniques developed here can be used in NICE. In this paper, we focus on algorithms for efficiently storing and locating trust information.

Another scheme [3] focuses on management and retrieval of trust-related data, and uses a single $\mathrm{p} 2 \mathrm{p}$ distributed database which stores complaints about individuals if transactions with them are not satisfactory. When an agent $p$ wants to evaluate 


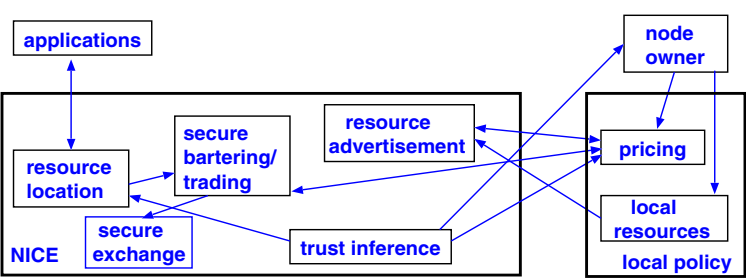

Fig. 1. NICE component architecture: the arrows show information flow in the system; each NICE component also communicates with peers on different nodes. In this paper, we describe the trust inference component of NICE.

trust for another agent $q$, it sends a query for complaint data which involves $q$, and decides $q$ 's trustworthiness with returned data, using a proposed formula. However, this system implicitly assumes that all participants are equally willing to share the communal data load, which may not be true in many p2p systems [4]. Such a system is also vulnerable to DoS attacks, as there is no preventative measure from inserting arbitrary amounts of complaints into the system.

PGP [18] is another distributed trust model that focuses on proving the identity of key holders. PGP uses user defined thresholds to decide whether a given key is trusted or not, and different introducers can be trusted at finite set of different trust levels. Unlike NICE, trust in PGP is only followed through one level of indirection; i.e. if $A$ is trying to decide the trust of $B$, there can be at most one person, $C$, in the trust path between $A$ and $B$. There are also a number of popular web sites, e.g. e-bay and Advogato (see www.advogato.org) that use trust models to serve their users. However, all data for these sites is stored at a trusted centralized database, which may not be ideal for open systems, and lead to the usual issues of scalability and single point of failure.

\section{OVERVIEW OF NICE}

In this section, we present a brief overview of the NICE platform. Our goal is to provide context for the distributed trust computation algorithms presented in Section IV. NICE is a platform for implementing cooperative distributed applications. Applications in NICE gain access to remote resources by bartering local resources. Transactions in NICE consist of secure exchanges of resource certificates. These certificates can be redeemed for the named (remote) resources. Noncooperative users may gain "free" access to remote resources by issuing certificates that they eventually do not redeem.

NICE provides a service API to end-applications, and is layered between the transport and application protocols. The NICE component architecture is presented in Figure 1. Applications interact with NICE using the NICE API, and issue calls to find appropriate resources. All of the bartering, trading, and redeeming protocols are implemented within NICE and are not exposed to the application. These sub-protocols share information within themselves and are controlled by the user using per-node policies. NICE peers are arranged into a signaling topology using our application-layer multicast protocol [5]. All
NICE protocol-specific messages are sent using direct unicast or are multicast over this signaling topology.

The NICE user identifier: Until now, we have used the term user and node in a generic manner. In NICE, each user chooses a PGP style identifier (See www.pgpi.org). The identifier includes a plaintext identification string and a public key. The key associated with a NICE identifier is used for signing resource certificates, for trading resources, and for assigning trust values.

It is important to note that neither the NICE identifier nor the associated key needs to be registered at any central authority; thus, even though NICE uses public keys, we do not require any form of a global PKI. Thus, NICE can be used to implement open $\mathrm{p} 2 \mathrm{p}$ applications without any centralized authority. Since there is no central registration authority in NICE, a single user can generate an arbitrary number of keys and personas. However, in NICE, pricing is coupled with identity (See Section III-A) and it is advantageous to maintain a single key per user and not to change keys frequently. This property makes NICE applications robust against a number of denial-of-service attacks that are possible on other $\mathrm{p} 2 \mathrm{p}$ systems.

In NICE, remote application actions are translated to local resource requests, and if feasible, the local resources are bartered for some resources at the remote node. (It is also possible to trade third-party resources instead of just bartering local resources). Obviously, not every remote request passes through NICE; instead, users barter and trade configurable units of resources (e.g. 100MB storage for 10 days, etc.). The resources are specified using a simplified version of the W3 RDF. NICE provides the following services:

- Resource advertisement and location

- Secure bartering and trading of resources

- Distributed "trust" valuation

Peers in NICE barter resources by exchanging "transaction" messages. A transaction message identifies sets of resources a principal is willing to barter. The integrity of a transaction message is ensured using a signed hash carried along with the message.

We use a Beaconing-based [14] scheme to scalably advertise and locate available resources. NICE uses a new fair exchange algorithm based on oblivious transfer protocols [10] to exchange resource certificates. This protocol assures that no party can gain a usable certificate without issuing a valid certificate; however, for the lack of space, we do not discuss the protocol in this paper. These resources certificates are eventually redeemed at the issuer nodes. Good nodes always redeem any certificate that they issue, while malicious nodes may choose not to. In the context of NICE, the specific goal of this paper is to develop algorithms to identify the nodes that issue good (eventually redeemable) certificates. Before we describe the trust inference component, we describe how the results of these schemes are used to price resources in NICE. 


\section{A. Pricing and Trading Policies}

The goal of the default policies in NICE is to limit the resources that can be consumed by cliques of malicious users. These policies work in conjunction with the trust computation which is used to identify the misbehaving nodes. In practice, NICE users may use any particular policy, and may even try to maximize the amount of resources they gain by trading their own resources. The primary goal of the default policies is to allow good users to efficiently form cooperating groups, and not lose large amounts of resources to malicious users. The pricing and trading policies are used to guard against users who issue spurious resource certificates using multiple NICE identities. We use two mechanisms to protect the integrity of the group:

\section{- Trust-based pricing}

In trust-based pricing, resources are priced proportional to mutually perceived trust. Assume trust values range between 0 and 1 , and consider the first transaction between Alice and Bob where the inferred trust value from Alice to Bob is $T_{\text {Alice }}(B o b)=0.5$, and $T_{B o b}($ Alice $)=1.0$. Under trust-based pricing, Alice will only barter with Bob if Bob offers significantly more resources than he gets back in return. Note however that as Bob conducts more successful transactions with Alice, the cost disparity will decrease. This policy is motivated by the observation that as Alice trades with a principal with lower trust she incurs a greater risk of not receiving services in return, which, in turn, is reflected in the pricing.

\section{- Trust-based trading limits}

In these policies, instead of varying the price of the resource, the policy varies the amount of the resource that Alice trades. For example, in the scenario with Alice and Bob, Alice may allow Bob to store $1 \mathrm{MB}$ of data at her host for one day, and gradually increase the storage and duration as she successfully redeems Bob's resource certificates. This policy assures that when trading with a principal with relatively low trust, Alice bounds the amount of resources she can lose.

\section{Distributed Trust Computation}

We assume that for each transaction in the system, each involved user produces a signed statement (called a cookie) about the quality of the transaction. For example, consider a successful transaction $t$ between users Alice and Bob in which Alice consumes a set of resources from Bob. After the transaction completes, Alice signs a cookie $c$ stating that she had successfully completed the transaction $t$ with Bob. Bob may choose to store this cookie $c$ signed by Alice, which he can later use to prove his trustworthiness to other users, including Alice ${ }^{2}$. As the system progresses, each transaction creates new cookies which are stored by different users.

\footnotetext{
${ }^{2}$ It is also possible for Alice to keep a record of this transaction instead of Bob. In this alternate model of trust information storage, users themselves store information about whom they trust, and can locally compute the trust of the remote nodes they know of. This model, however, is susceptible to a denial of service attack that we describe later in this section.
}

(Clearly, cookies have to be expired or otherwise discarded; the algorithms we present later in this section require constant storage space.)

We will describe the trust inference algorithms in terms of a directed graph $T$ called the trust graph. The vertices in $T$ correspond exactly to the users in the system. There is an edge directed from Alice to Bob if and only if Bob holds a cookie from Alice. The value of the Alice $\rightarrow$ Bob edge denotes how much Alice trusts Bob, and depends on the set of Alice's cookies Bob holds. Note that each transaction in the system can either add a new directed edge in the trust graph, or relabel the value of an existing edge with its new trust value.

Assume that a current version of the trust graph $T$ is available to Alice, and suppose Alice wishes to compute a trust value for Bob. If Alice and Bob have had prior transactions, then Alice can just look up the value of Alice $\rightarrow$ Bob edge in $T$. However, suppose Alice and Bob have never had a prior transaction. Alice could infer a trust value for Bob by following directed paths (ending at Bob) on the trust graph.

\section{A. Inferring Trust on the Trust Graph}

Consider a directed path $A_{0} \rightarrow A_{1} \rightarrow \ldots \rightarrow A_{k}$ on $T$. Each successive pair of users have had direct transactions with each other, and the edge values are a measure of how much $A_{i}$ trusts $A_{i+1}$. Given a such a path, $A_{0}$ could infer a number of plausible trust values for $A_{k}$, including the minimum value of any edge on the path or the product of the trust values along the path; we call these inferred trust values the strength of the $A_{0} \rightarrow A_{k}$ path. The inference problem is somewhat more difficult than computing strengths of trust paths since there can be multiple paths between two nodes, and these paths may share vertices or edges. Centralized trust inference is not the focus of this paper (or of our work), but it is important to use a robust inference algorithm. We have experimented with different inference schemes, and we describe two simple but robust schemes. In the following description, we assume A (Alice) has access to the trust graph, and wants to infer a trust value for $\mathrm{B}$ (Bob):

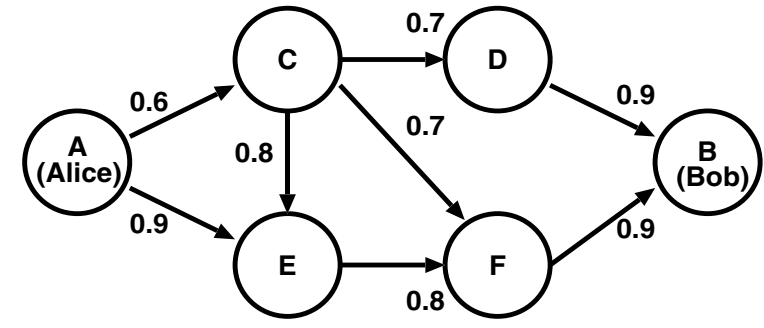

Fig. 2. Example trust graph: the directed edges represent how much the source of edge trusts the sink.

- Strongest path: Given a set of paths between Alice and Bob, Alice chooses the strongest path, and uses the minimum trust value on the path as the trust value for Bob. The strength of a path can be computed as the 
minimum valued edge along the path or the product of all edges along a path. Given the trust graph, this trust metric can easily be computed using depth-first search. In the example shown in Figure 2, we use the min. function to compute the strength of a path. In this example, the strongest path is $A E F B$, and Alice infers a trust level of 0.8 for Bob.

- Weighted sum of strongest disjoint paths: Instead of choosing only the strongest path, Alice could choose to use contributions from all disjoint paths. The set of disjoint paths is not unique, but the set of strongest disjoint paths (modulo equi-strength paths) is and can be computed using network flows with flow restrictions on vertices. Given the set of disjoint paths, Alice can compute a trust value for Bob by computing the weighted sum of the strength of all of the strongest disjoint paths. The weight assigned to the Alice $\rightarrow X \rightarrow \ldots \rightarrow$ Bob path is the value of the Alice $\rightarrow X$ edge (which represents how much Alice directly trusts X). In the example in Figure 2, $A C D B$ is the other disjoint path (with strength 0.6), and the inferred trust value from Alice to Bob is 0.72 .

Both these algorithms are robust in the sense that no edge value is used more than once, and trust values computed are always upper-bounded by the minimum trust on a path. Before any of these local algorithms can be used, the trust graph has to be realized in a scalable manner, and (edge) values have to be assigned to cookies. Note that in order to infer trust for Bob, Alice does not need the entire trust graph, but only needs the set of paths from her to Bob. In the rest of this section, we describe schemes to store the trust graph and to produce sets of paths between users in a completely decentralized manner over an untrusted infrastructure. We begin with a discussion of different techniques for assigning cookie values, and describe our distributed path discovery protocol in Section IV-C.

\section{B. Assigning Values to Cookies}

Ideally, after each transaction, it would be possible to assign a real number in the $[0,1]$ real-valued interval to the quality of a transaction and assign this as the cookie value. In some cases, transactions can be structured such that this indeed is possible: e.g. assume that Alice transcodes and serves a $400 \mathrm{Kbps}$ video stream to Bob at $128 \mathrm{Kbps}$, and according to a prior agreement, Bob signs over a cookie of value 0.75 to Alice. The same transaction may have resulted in a cookie of value 0.9 if Alice had been able to serve the stream at $256 \mathrm{Kbps}$. In many cases, however, it is not clear how to assign real-valued quality metrics to transactions. For example, in the previous example, Alice could claim that she did serve the stream at $256 \mathrm{Kbps}$, while network congestion on Bob's access link caused the eventual degradation of the quality to $128 \mathrm{Kbps}$. It is, in fact, easy to construct cases when it is not easily feasible to check the quality of service. In most cases, however, we believe it is somewhat easier to assign a $\{0,1\}$ value to a transaction, i.e. either the transaction was successful, or it was not. In the previous example, Bob and Alice negotiate a threshold rate (say 64Kbps) at which point he considers the entire transaction successful, and assigns a 1-valued cookie to Alice regardless of whether the data was delivered at $64.5 \mathrm{Kbps}$ or $400 \mathrm{Kbps}$. Further, for many transactions, such as streaming media delivery, it is possible for one party to abort the transaction if the initial service quality is not beyond the 0 -value threshold.

In the rest of this paper, we assume that cookies are assigned values on the $[0,1]$ interval. However, it is possible to assign arbitrary labels to cookies, and to conduct arbitrary policybased searches as long as the requisite state is kept at each user. For example, it is possible to construct a system where cookies take one of four values (e.g., "Excellent", "Good", "Fair", and "Poor"), and users search for "Excellent"-valued cookies that are less than one week old. All of the NICE path enumeration and inference schemes work correctly as long as cookies have a comparable value, regardless of how users assign these values, and what range these values take.

\section{Distributed Trust Inference: Basic Algorithm}

In this section, we describe how users locate trust information about other users in our system. This distributed algorithm proceeds as follows: each user stores a set of signed cookies that it receives as a result of previous transactions. Suppose Alice wants to use some resources at Bob's node. There are two possibilities: either Alice already has cookies from Bob, or Alice and Bob have not had any transactions yet. (There is yet a third possibility in which Alice has discarded cookies from Bob, but we assume that this case is equivalent to Alice having no cookies from Bob). In the case Alice already has cookies from Bob, she presents these to Bob. Bob can verify that these indeed are his cookies since he has signed them. Given the cookies, Bob can now compute a trust value for Alice.

The more interesting case is when Alice has no cookies from Bob. In this case, Alice initiates a search for Bob's cookies at nodes from whom she holds cookies. Suppose Alice has a cookie from Carol, and Carol has a cookie from Bob. Carol gives Alice a copy of her cookie from Bob, and Alice then presents two cookies to Bob: one from Bob to Carol, and one from Carol to Alice. Thus, in effect, Alice tells Bob, "You don't know me, but you trust Carol and she trusts me!" In general, Alice can construct multiple such "cookie paths" by recursively searching through her neighbors. In effect, Alice floods queries for Bob's cookies along the cookie edges that terminate at each node, starting with her own node. After the search is over, she can present Bob with a union of directed paths which all start at Bob and end at Alice. Note that these cookie paths correspond exactly to the union of directed edges on the trust graph which we used for centralized trust inference. Thus, given this set of cookies, Bob can use any centralized scheme to infer a trust value for Alice.

This basic scheme has several desirable properties:

- If Alice wants to use resources at Bob, she has to search for Bob's cookies. This is in contrast with the analogous scheme in which nodes themselves keep records of their previous transactions. Under such a setting, if Bob did not 

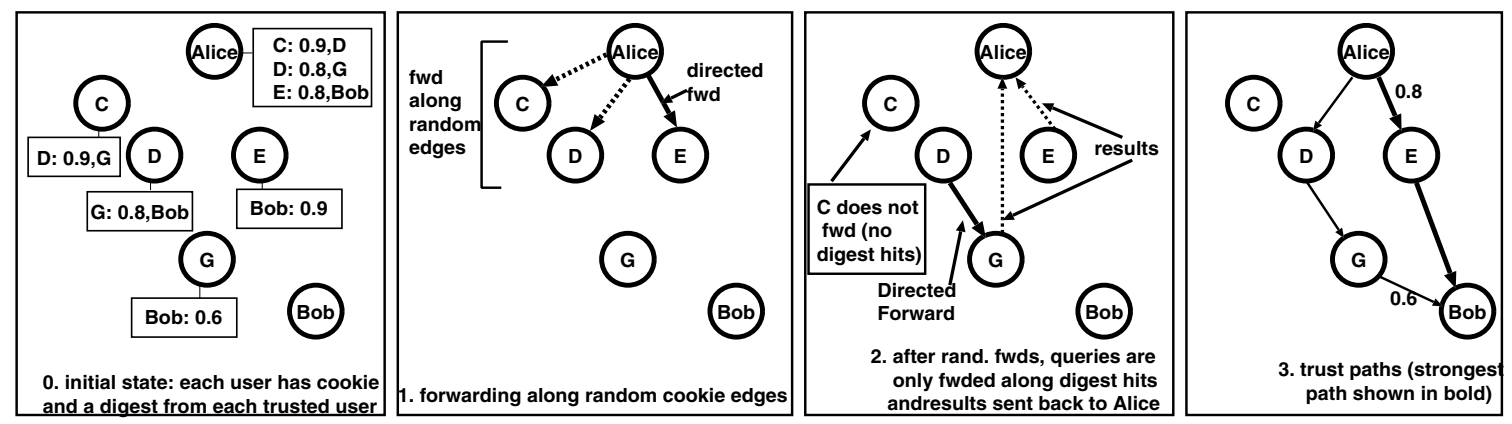

Fig. 3. Different stages in the operation of the search protocol. Edges in this figure represent message flow. It is important to note that corresponding edges in the trust graph point in the opposite direction.

know Alice, he would have to initiate a search for Alice through nodes he trusted. A malicious user Eve could mount an easy denial-of-service attack by continuously asking other nodes to search for Eve's credentials.

In our system, nodes forward queries on behalf of other nodes only if they have assigned them a cookie, and thus, implicitly trust them to a certain extent.

- Alice stores cookies which are statements of the form "X trusts Alice". Thus, Alice only devotes storage to items that she can use explicitly for her own benefit, and thus, there is a built-in incentive in the system to store cookies. In fact, if Bob assigns a low-value cookie for Alice, she can discard this cookie since this is, in effect, a statement that says Bob does not trust Alice. In general, users store the cookies most beneficial to their own cause, and do not forward messages on behalf of users they do not trust.

- The transaction record storage in the system is completely distributed, and if two nodes conduct a large number of spurious transactions, only they may choose to hold on to the resultant state. In contrast, in a centralized transaction store, these nodes could easily mount a denialof-service attack by overwhelming the transaction store with spurious transaction records.

\section{Refinements}

While the flooding-based scheme we have described is guaranteed to find all paths between users and has other desirable properties, it is not a complete solution. Flooding queries is rather an inefficient usage of distributed resources, and as pointed out before, malicious nodes can erase all information of their misdeeds simply by throwing away any low valued cookies they receive. We next describe three refinements to the basic scheme.

1) Efficient Searching: The recursive flooding procedure described above does find all cookies that exist for a given principal. However, it is extremely inefficient, since it visits an exponentially growing number of nodes at each level. Further, unless the flooding is somehow curtailed, e.g. by using duplicate suppression or by using a time-to-live field in queries, some searches may circulate in the system forever.
It is obvious to consider using a peer-to-peer search structure, such as Chord, to locate cookies. However, this is not possible since in NICE we do not assume the existence of anything more sophisticated than plain unicast forwarding. NICE is the base platform over which other protocols, such as Chord, can be implemented. The NICE protocols are much like routing protocols on the Internet: they cannot assume the existence of routing tables etc., and must be robust against packet loss and in the case of NICE, against malicious nodes. Thus, we must employ other mechanisms to make the cookie searches more efficient.

Instead of following all paths corresponding to all cookies, we only forward a query to a number of nodes (typically 5) from any one node. However, if we chose the forwarding nodes at random, we would still have an exponential search, albeit with a smaller base! Instead, we add the following extension to our base protocol: whenever node receives a cookie from some other node, it also receives a digest of all other cookies at the remote node. Since, in our implementations, the number of cookies at each node is quite small (typically around 40 for a 2048 node system), this digest can be encoded using around less than 1000 bits in a Bloom filter ${ }^{3}$ [7]. Thus, the storage space required for the digests are trivial (around 128 bytes), but they allow us to direct the search for specific cookies with very high precision. The idea of using digests for searches has been used previously, e.g. in lookaround caching [6] and summary caches [8]. It is, in fact, a base case of probabilistic search using attenuated Bloom filters [12]; in our experiments, we found that we did not need to use full attenuated Bloom filters - only one level of filters was sufficient. Lastly, each node also keeps a digest of recently executed searches and uses this digest to suppress duplicate queries.

In our implementation, when choosing nodes to forward to, we always choose nodes whose digests indicate they have the cookie for which we are searching. However, it is possible that there are no hits in any digest at a node; in this case, we once again choose nodes to forward to uniformly at random. However, we only forward to randomly chosen nodes if the

\footnotetext{
${ }^{3}$ Such a filter, with only eight hash functions, would have a false positive rate of $3.16 \times 10^{-5}$.
} 
query is within a pre-determined number of hops away from the query source. Thus, in the final version of the search, a query spreads from the source, possibly choosing nodes at random, but the flooding is quickly stopped unless there is a hit in a next-hop digest.

Example: Before we describe other extensions to the base protocol, we illustrate the digest-based search procedure with an example (corresponding to Figure 3). Alice wants to use resources Bob has, but does not have cookie from him. She initiates a search for a cookie path to Bob. In Figure 3-0 we show the initial state of cookies and digests at each user, e.g. Alice has a cookie of value 0.9 from $\mathrm{C}$, and her digest from $\mathrm{C}$ shows that $\mathrm{C}$ has a cookie from $\mathrm{D}$. For this example, we assume the search outdegree is 3 , and the random flooding hop limit is 1 . Alice first sends a query not only to nodes with digest hit (e.g. $E$ ), but also to random nodes (e.g. $C$ and $D$ ) as illustrated in Figure 3-1. After receiving the query, $E$ finds Bob's cookie and returns the query to Alice. When $C$ receives the query, he finds that none of his neighbors have a digest hit for Bob, so does not forward the query further. On the other hand, $D$ does forward the query to $G$ (Figure 3-2) who has a digest hit for Bob, and $G$ returns the query to Alice with the cookie she received from Bob. Figure 3.3 shows two paths Alice finds, with the strongest path in bold.

2) Negative Cookies: A major flaw with the original scheme is that low-valued transactions are potentially not recorded in the system. Consider the following scenario: Eve uses a set of Alice's resources, but does not provide the negotiated resources she promised. In our original scheme, Alice would sign over a low valued cookie to Eve. Eve would have no incentive to keep this cookie and would promptly discard it, thus erasing any record of her misdeed.

Instead, Alice creates this cookie and stores it herself. It is in Alice's interest to hold on to this cookie; at the very least, she will not trust Eve again as long as she has this cookie. However, these "negative cookies" can also be used by users who trust Alice. Suppose Eve next wants to interact with Bob. Before Bob accepts a transaction with Eve, he can initiate a search for Eve's negative cookies. This search proceeds as follows: it follows high trust edges out of Bob and terminates when it reaches a negative cookie for Eve. In effect, the search returns a list of people whom Bob trusts who have had negative transactions with Eve in the past. If Bob discovers a sufficient set of negative cookies for Eve, he can choose to disregard Eve's credentials, and not go through with her proposed transaction. It is important to note that Bob only initiates a negative cookie search when Eve produces a sufficient credible set of credentials; otherwise, Bob is subject to a denial of service attack where he continuously searches for bad cookies.

In implementation, we keep a set of digests for negative cookies as well, but perform Bloom filter directed searches for these negative cookies only on neighboring nodes.

3) Preference Lists: In order to discover potentially "good" nodes efficiently, each user keeps a preference list. For each user, the nodes in her preference list consists of a set of other users that she has not conducted a transaction with yet. However, these nodes are preferred since the owner of the preference list believes they may be potentially high trust peers. Nodes are included in a preference list as follows: suppose Alice conducts a successful cookie search for Bob, and let $P$ be the cookie path that is discovered between Alice and Bob. Alice adds all users in $P$ who have very high trust value (1.0 in our implementation) to her preference list. Obviously, only users for whom Alice does not have transaction records are added to her preference list.

In summary, the NICE distributed trust valuation algorithm works as follows:

Nodes that request resources present their credentials to the resource owner. Each credential is a signed set of certificates which originate at the resource owner. Depending on the set of credentials, the resource owner may choose to conduct a reference search. The trust ultimately computed is a function of both the credentials, and of the references.

There are a number of other pragmatic issues pertaining to cookies that we address in NICE. These include cookie revocations, cookie time limits, etc. Due to space constraints, we do not describe these further in this paper.

\section{Results}

In this section, we present results from our simulations of the trust inference algorithm proposed in Section IV. In all our results, we use the minimum cookie value as path strength, and use the strongest path strength as the inferred trust between users. We have experimented with other functions as well, and the results from this simple inference function are representative. Each search carries with it the minimum acceptable strength, and searches stop if no cookies of the minimum acceptable value are present at the current node. Using the minimum cookie value as the strength measure (instead of product of cookie values) consumes up to an order of magnitude more resources in the network and represent a worst-case scenario for our schemes.

We divide our results into two parts. First, we analyze the cost of running the path search algorithm in terms of storage and run time overhead. The storage cost is entirely due to the caching of various positive and negative cookies; the run-time overhead comes from the number of nodes that are visited by each query, and the computation cost for forwarding a query. The computation cost of forwarding each query is negligible: we have generated a few random numbers, compute eight MD5 hash functions, and check eight bits in a 1000-bit Bloom filter. In these experiments, the digests were always fresh. We did not simulate updating of the digest, but we believe a periodic soft-state refreshing algorithm will work adequately. The main overhead of the search algorithm comes in terms of the number of messages and number of nodes visited on the network. The bandwidth consumed by the searches is proportional to the number of nodes visited, and we report this metric in the results that follow. In the second part of our results (Section VB), we show that our trust inference schemes do indeed form 


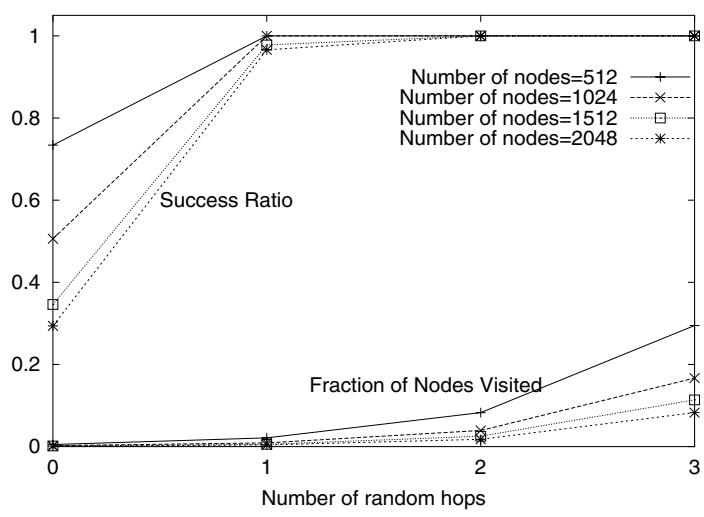

Fig. 4. Success ratio and no. of nodes visited ( 40 cookies at each node).

robust cooperative groups, even in large systems with a large malicious cliques and with small fractions of good nodes. We begin with an analysis of the scalability and overhead of our path searches.

\section{A. Scalability}

In this first set of results, we simulate a stable system consisting of only good users. Thus, we assume that all users implement the entire search protocol correctly. Before the simulations begin, we fill the (good) cookie cache of each user by adding cookies from other users chosen uniformly at random. Each query starts at a node $s$ chosen uniformly at random and specifies a search for cookies of another node $t$ chosen uniformly at random. In the next section, we will show how long the system takes to converge starting from no cookies in the system, and how robust groups are formed when there are malicious users in the system.

In our first experiment, we fix the number of good cookies at each user to 40 . The cookie values are exponentially distributed between $[0,1]$, with a mean of $0.7^{4}$. Next we conducted 500 different searches for cookies of value at least 0.85 , where the search outdegree at each node is set to 5 . In Figure 4, we plot the average success ratio and the average fraction of nodes in the system visited by the searches. The $x$-axis in the plot corresponds to the number of hops after which random forwards were not allowed, and the search proceeded only if there was a hit in a Bloom filter. There are four curves in the figure, each corresponding to a different system size, ranging from 512 users to 2048 users. From the figure, it is clear that only one hop of random searching is enough to satisfy the vast majority of queries, even with large system sizes. It is interesting to note that even when the system size increases, the average number of nodes visited remain relatively constant. For example, the average number of nodes visited with 2 hop random searches range from 42.4 (for a 512 node system) to 36.2 (for the 2048 node system). Thus, the

\footnotetext{
${ }^{4}$ It is not clear how cookie values should be distributed. We have also experimented with uniformly distributed cookie values with similar results.
}

\begin{tabular}{c|cccccccc} 
& \multicolumn{2}{|c}{$\mathrm{K}=3$} & \multicolumn{2}{c}{$\mathrm{K}=5$} & \multicolumn{2}{c}{$\mathrm{K}=7$} & \multicolumn{2}{c}{$\mathrm{K}=20$} \\
thrsh. & \# N & \# P & \# N & \# P & \# N & \# P & \# N & \# P \\
\hline .8 & 14.5 & 4.2 & 37.5 & 10.7 & 71.1 & 20 & 499.1 & 132.5 \\
.85 & 14.6 & 3.6 & 36.2 & 8.7 & 68.7 & 16.5 & 380.6 & 88.3 \\
.9 & 14.6 & 2.9 & 35.1 & 6.8 & 66.0 & 12.8 & 222.9 & 41.5 \\
.95 & 15.7 & 2.0 & 33.2 & 4.2 & 55.9 & 7 & 89.2 & 11.2
\end{tabular}

TABLE I

EFFECT OF CHANGING OUTDEGREE (K)

\begin{tabular}{c|cccccc} 
& \multicolumn{2}{|c}{$\mathrm{C}=20$} & \multicolumn{2}{c}{$\mathrm{C}=40$} & \multicolumn{2}{c}{$\mathrm{C}=102$} \\
thresh. & \# N & \# P & \# N & \# P & \# N & \# P \\
\hline 0.8 & 34.4 & 2.9 & 37.5 & 10.7 & 32.8 & 23.6 \\
0.85 & 34.7 & 2.4 & 36.2 & 8.7 & 37.3 & 25.4 \\
0.9 & 34.7 & 1.9 & 35.1 & 6.8 & 40.8 & 25.4 \\
0.95 & 28.6 & 1.4 & 33.2 & 4.2 & 41.9 & 21.5
\end{tabular}

TABLE II

EFFECT OF CHANGING NUMBER OF COOKIES STORED (C)

search scheme scales extremely well with increasing system size. As we show next, the success ratio and the number of nodes visited depend almost entirely on the number of cookies held at each node, and the outdegree of each search.

In Table I, we fix the number of nodes to 2048 and show the effect of changing the search outdegree. Each row shows searches corresponding to a different minimum threshold ranging from 0.8 to 0.95 . Each node holds 40 cookies, the average cookie value is still fixed at 0.7 , and the number of random hops is set to 2 . In the table, $\# N$ is the average number of nodes visited by a query and \#P denotes the number of paths found on average. As expected, the number of nodes visited increases as the search threshold decreases, and also as the outdegree increases. In all cases, as the search threshold increases, the number of distinct paths found decreases. In Table II, we show the effects of changing the number of cookies at each node. These experiments are conducted using the same parameters, except the outdegree is fixed at 5 . With small numbers of cookies and high thresholds, searches do result in no paths being found. In Table II, the 0.9 and 0.95 threshold searches had $10 \%$ and $42 \%$ unsuccessful queries respectively; all other searches returned at least one acceptable path. In our simulator, when a search returns no acceptable paths, we retry the search once more with a different random seed. The numbers for nodes visited in the results above include visits during the retries, and account why the number of nodes visited do not decrease when the search threshold is increased.

In our system, there is a clear tradeoff between how much state individual nodes store (number of cookies) and the overhead of each search (fraction of nodes visited). Note that unlike in systems such as [3], users in our system do not gain by storing fewer cookies since this effectively decreases their own expected trust at other nodes. There is a built-in incentive for users to store more cookies, which, in turn, increases search efficiency. Users may choose to store a large number 
of cookies but not forward searches on behalf of others. We comment on this issue when we discuss different models of malicious behavior in the next section. Lastly, we note that it is possible to further increase the efficiency of the searches by adjusting the two search parameters - outdegree and number of random hops- based on the threshold and results found. Such a scheme will minimize the number of nodes visited for "easy" searches (low search threshold), and find better results for searches with high thresholds. We have not implemented this extension yet.

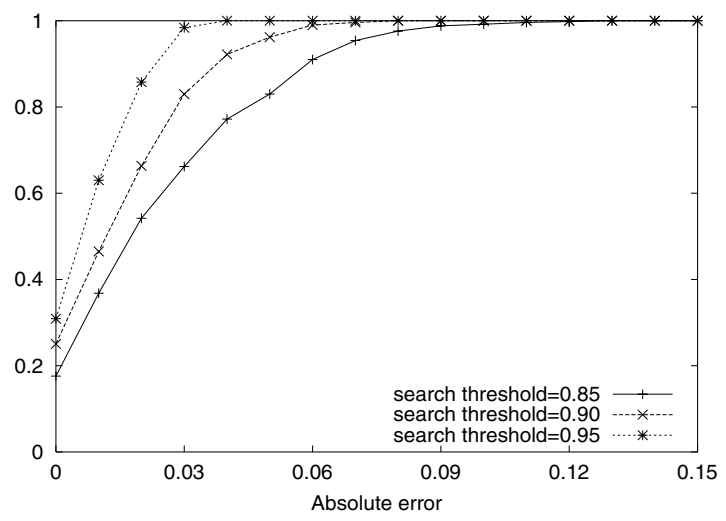

Fig. 5. CDF of errors in results (40 cookies at each node, outdegree set to 5) with varying thresholds.

The previous two results have shown that the number of cookies and search outdegree provides an effective mechanism to control the overhead of individual searches. However, in each case, we have only shown that each search returns a set of results - it is possible that the searches find paths that are above the search threshold, but are not the best possible paths. For example, suppose that a search for threshold set to .85 returns a path with minimum cookie value .90 . This is an acceptable result; however, there may be a better path that the search missed (e.g. with minimum cookie value .95). In this case, the best path returned had an absolute error of .05. To quantify the quality of the found paths, we plot the absolute error in the paths returned by our searches as compared to an optimal search (full flooding). In Figure 5, we plot the CDF of the absolute error for the best path that we find versus the best possible cookie path as the search threshold is changed. The higher threshold searches have a less possible absolute margin of error, and thus produce the best paths. However, very high threshold searches are also more likely to produce no results at all.

\section{B. Robustness}

In the rest of the results, we analyze two components of the system: how long it takes for the system to stabilize, and how well our system holds up against malicious users. Modeling malicious users is an important open research question: one for which we do not provide any particular insights in this paper. Instead, we use a relatively simplistic user model with three different types of users:

- Good users: Good users always implement the entire protocol correctly. If a good user interacts with another good user, then the cookie value assigned is always 1.0. Good users do not know the identity of any other good (or otherwise) users at the beginning of the simulation.

- Regular users: Regular users always implement the entire protocol correctly; however, when a regular user interacts with another user, transactions result in cookie value that range exponentially between 0.0 and 1.0 , with a mean of 0.7 . Regular users also do not know of any other users when the simulations begin.

- Malicious users: All malicious users form a cooperating clique before the simulation begins. Further, each malicious user always reports implicit trust (cookie value 1.0) for every other malicious user. Once a malicious user interacts with a non-malicious user, the malicious user produces either a high trust value cookie (between 0.9 and 1.0) or a very low value cookie (between 0.0 and 0.1 ). The probability of a transaction failing is uniform and random, and the average is a simulation parameter.

In the experiments that follow, each user stores 40 good cookies and 40 negative cookies. Each experiment was conducted with 512 users, of which 24 were good.

At each time step in the simulation, a user (say Alice), is chosen uniformly at random. Alice selects another user (say Bob) from her preference list to initiate a transaction with. If Alice's preference list is empty, she chooses the user Bob uniformly at random. This transaction commences if Bob can find at least one path of strength at least 0.85 between himself and Alice and if Bob cannot locate a negative cookie for Alice. If the transaction between Alice and Bob cannot proceed, Alice tries her transaction with a different user. After two unsuccessful tries, Alice chooses a random user Carol and the simulator allows a transaction without checking Alice's credentials. Cookies are flushed from a user's cookie cache using the following rule: cookies of value 1.0 are not flushed; other cookies are discarded with uniform probability.

In the first result, we only consider good and regular users (there were 488 regular users and 24 good users in this experiment). In Figure 6, we plot the fraction of transactions between good users and the fraction of paths between good users. The $x$-axis shows the total number of transactions in which at least one party was a good node. (We choose this measure as the $x$-axis because in a real system, malicious nodes can fabricate any number of spurious transactions, and the only transactions that matter are the ones involving good nodes). The effect of the preference lists is clear from the plot: even though there is a less than $5 \%$ chance of a good node interacting with another good node, there is a path between any two good nodes within 1500 transactions. By 2500 total transactions, the majority of which were between good nodes and regular nodes, all good nodes have cookies from all other good nodes, and the robust cooperative group has formed. This 
good clique will not be broken unless a good node turns bad, since 1.0 valued cookies are not flushed from the system.

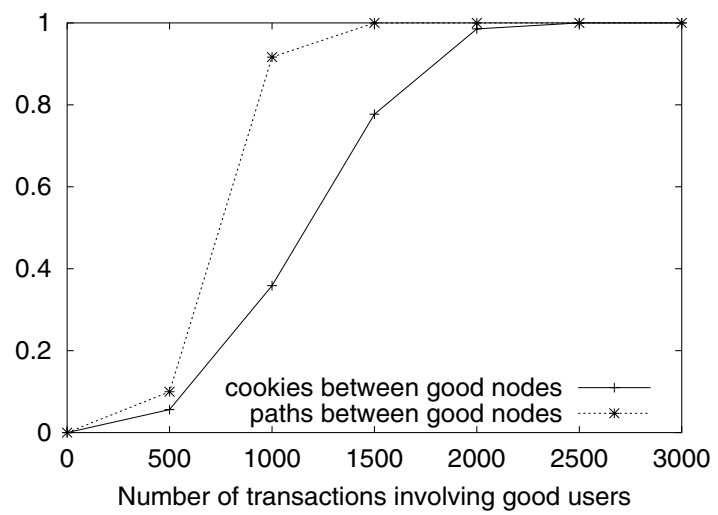

Fig. 6. System initialization with good and regular users.

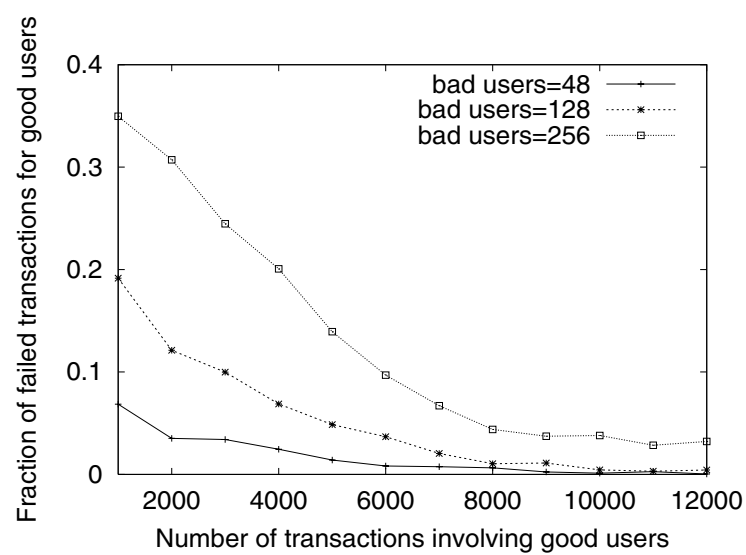

Fig. 7. Failed transactions for good users ( 40 cookies at each node, 512 nodes total, 0.5 transaction failure probability).

In the next set of results, we introduce bad (malicious) nodes. Figure 7 illustrates the fraction of failed transactions involving good nodes normalized by the total number of transactions involving good nodes. The curves in the figure show failed transactions involving regular nodes and bad nodes for varying numbers of bad nodes in the system, averaged over 1000 transaction intervals. For these results, we define failed transactions as those that produce a cookie of value less than 0.2 , and the probability that a transaction fails is 0.5 . In the beginning of the simulation, the number of failed transactions are proportional to the number of bad users in the system. However, for all bad user populations, the good users identify all bad users and the number of good-bad transactions approaches zero. The effect of the preference lists is again apparent in this experiment: recall that all bad nodes always report 1.0 trust for other bad nodes. Thus, bad nodes rapidly fill the preference lists of good nodes, but are quickly identified as malicious.

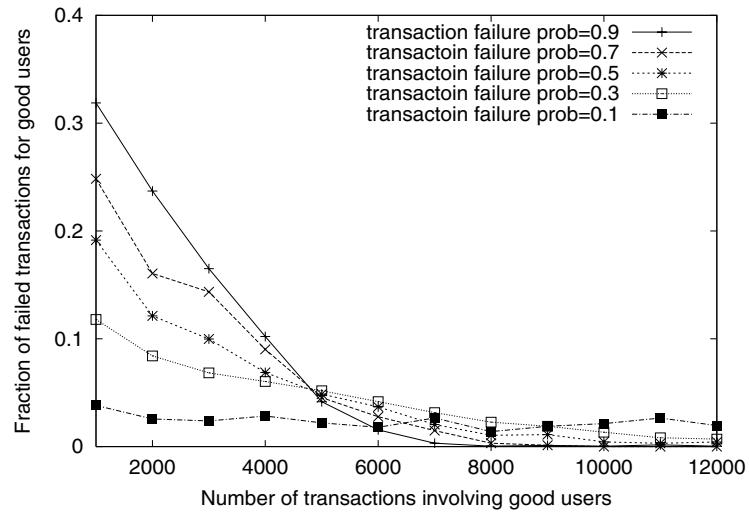

Fig. 8. Failed transactions with varied failure probability (40 cookies at each node, 512 nodes total, 128 malicious nodes)

Lastly, we report the result when we varied the failure probability of transactions with malicious users (Figure 8). As expected, when the probability of failure is set high, malicious nodes are identified easily and transactions with them are quickly avoided. More specifically, when the failure probability is 0.9 , there are many failed transactions in the early stage (again, due to preference list). However, the number abruptly drops, and there are virtually no failures after 7000 transactions. On the other hand, in case of lower failure probabilities, we can see the number of transactions required to identify bad nodes increases. In particular, we note that the number of failed transactions does not change much over time when the failure probability is 0.1 , and that bad nodes are not easily identified. However, such behavior (good for $90 \%$ of the time) itself is not very harmful and their average trust values are even higher than regular nodes. We can also observe from this simulation result that in all cases, the number of failed transactions eventually falls below $5 \%$.

In our experiments, good users do not preferentially interact with other good users (as would be expected in a real system). Instead, if their preference lists are empty, they pick a random user to interact with. Recall that there are an order of magnitude more regular users in the system than good users. Thus, good users continue to interact with regular users and approximately $5 \%$ of good user transactions result in failures (not shown in Figure). In a deployed system, the fraction of failed transactions would be much smaller, since the vast majority of transactions initiated by good users would involve other good users.

It is important to note that even with many malicious users, a robust cooperative group emerges in our system. This property is true, regardless of the number of positive or negative cookies good users keep, as long as good users can choose random other users to conduct transactions with. Otherwise, bad cliques can stop good users from ever communicating with another good user. However, as long as bad users cannot stop whom good users talk to, a cooperative group emerges. The good users eventually find and keep state from other 
good users, and this state cannot be displaced by malicious users. Obviously, the number of transactions required for good cliques to form depends on the number of malicious nodes in the system, but good users rapidly find other good users by using their preference lists. It is possible for a malicious node to infiltrate good cliques for prolonged periods, but as these bad nodes conduct transactions that fail, the negative cookies will cause these users to be rapidly discarded from the good clique.

Other user models: We have varied other parameters in our experiments, and present a summary of our findings. We experimented with a different malicious node model in which the bad nodes do not forward queries from non-malicious nodes. The results for this model were not appreciably different from the model we have used in our results. We have also experimented with models in which bad users actively publish negative cookies for good users. As these users are identified as bad by the good users, these negative cookies are rendered useless. Lastly, we note that our good user model is probably too simplistic. Even good users may be involved in failed transactions, possibly due to no fault of their own. However, we believe our results will still hold as long as there is a definite and marked difference between the behavior of good and bad users.

\section{Summary AND CONCLUSIONS}

The main contribution of this paper is a low overhead trust information storage and search algorithm which is used in the NICE system to implement a range of trust inference algorithms. Our scheme is unique in that the search and inference performance for the whole group increases as users store more information that is explicitly beneficial for their own cause. We have presented a scalability study of our algorithms, and have shown that our technique is robust against a variety of attacks by malicious users. We believe techniques presented in this paper are a crucial piece for building large peer-to-peer systems for deployment over the Internet.

\section{REFERENCES}

[1] Gnutella home page. http://gnutella.wego.com.

[2] Alfarez Abdul-Rahman and Stephen Hailes. Supporting Trust in Virtual Communities. In Proceedings Hawaii International Conference on System Sciences 33, 2000.

[3] Karl Aberer and Zoran Despotovic. Managing trust in a Peer-2Peer information system. In Henrique Paques, Ling Liu, and David Grossman, editors, Proceedings of the Tenth International Conference on Information and Knowledge Management (CIKM-01), pages 310317, New York, November 5-10 2001. ACM Press.

[4] E. Adar and B.A. Huberman. Free riding on gnutella. Technical report, Xerox PARC, August 2000.

[5] S. Banerjee, B. Bhattacharjee, and C. Kommareddy. Scalable application layer multicast. In Proc. ACM Sigcomm, August 2002.

[6] Samrat Bhattacharjee, Ken Calvert, and Ellen Zegura. Self-organizing wide-area network caches. In IEEE Infocom'98, 1998.

[7] Burton H. Bloom. Space/time trade-offs in hash coding with allowable errors. Communications of the Association for Computing Machinery, 13(7):422-426, 1970.

[8] Li Fan, Pei Cao, Jussara Almeida, and Andrei Z. Broder. Summary cache: A scalable wide-area web cache sharing protocol. Proc. SIGCOMM'98, 28(4):254-265, September 1998.

[9] S. Marsh. Formalising Trust as a Computational Concept. PhD thesis, University of Sterling, 1994.

[10] M. Naor and B. Pinkas. Efficient Oblivious Transfer Protocols. In Proceedings of SODA, January 2001.

[11] Sylvia Ratnasamy, Paul Francis, Mark Handley, Richard Karp, and Scott Shenker. A scalable content addressable network. In Proceedings of the ACM SIGCOMM 2001 Technical Conference, 2001.

[12] Sean C. Rhea and John Kubiatowicz. Probabilistic location and routing. In Proceedings of INFOCOM 2002, 2002.

[13] Antony Rowstran and Peter Druschel. Pastry: Scalable, distributed object location and routing for large-scale peer-to-peer systems. In Proceedings of the 18th IFIP/ACM International Conference on Distributed Systems Platforms (Middleware 2001), 2001.

[14] Narendar Shankar, Christopher Komareddy, and Bobby Bhattacharjee. Finding Close Friends over the Internet. In Proceedings of Internation Conference on Network Protocols, November 2001.

[15] Ion Stoica, Robert Morris, David Karger, M. Frans Kaashoek, and Hari Balakrishnan. Chord: A scalable peer-to-peer lookup service for internet applications. In Proceedings of the ACM SIGCOMM '01 Conference, San Diego, California, August 2001.

[16] Bin Yu and Munindar P. Singh. A social mechanism of reputation management in electronic communities. In Proceedings of Fourth International Workshop on Cooperative Information Agents, 2000.

[17] S. Q. Zhuang, B. Y. Zhao, A. D. Joseph, R. Katz, and J. Kubiatowicz. Bayeux: An architecture for scalable and fault-tolerant wide-area dat a dissemination. In NOSSDAV 2001, 2001.

[18] P. Zimmermann. Pretty good privacy user's guide. Distributed with PGP software, June 1993. 